\title{
Data Management by ZEISS
}

Martin Kuttge

\section{ZEISS Research Microscopy Solutions, München, Bayern, Germany}

Data management in core facilities is becoming more important as complexity of sample investigation increases. More and more users move between different systems of a core facility to investigate their samples to the full extend and to leverage the full potential of analytical possibilities. As a result, they are facing a data overload dilemma trying to combine often heterogenous data and to visualize, analyze and document the variety of results. Keeping the data connected and accessible during a workflow becomes critical, even more so if several users are involved.

As a company with a broad portfolio of instruments ZEISS has been following this trend with our customers and in this talk we will present recent developments by ZEISS in the field of data management and data fusion which help microscope users in core facilities in their daily workflows in their way to a truly connected lab.

The ZEN Connect module integrated in the ZEN microscope control software allows a seamless fusion of data from different instruments. The combined data can then be used for guided navigation and comprehensive documentation of the results. At the same time, ZEN Connect is keeping a track of the data and presents it in well structured projects so that users can easily reconstruct from which instrument certain data originates and when it was acquired.

Storing and retrieving the data from a central repository can be achieved using the ZEN Data Storage image database. It allows a central server-based access and sharing of data as well as protocols and templates and is directly integrated into ZEN. The ZEN Data Storage database is complemented with the ZEN Data Explorer, a web browser and app-based solution to view and annotate data remotely and away from your microscopes.

It is important to note that ZEISS is opening these solutions also to customers who would like to store and visualize data and images from other vendors by allowing an open import and storage interface for the ZEISS data management solutions. 
https://doi.org/10.1017/S1431927620022473 Published online by Cambridge University Press 